\title{
Fourier series of finite products of Bernoulli and Genocchi functions
}

Taekyun Kim ${ }^{1,2}$, Dae San Kim³ ${ }^{3}$ Gwan-Woo Jang ${ }^{1}$ and Jongkyum Kwon ${ }^{4 *}$

"Correspondence:

mathkjk26@gnu.ac.kr

${ }^{4}$ Department of Mathematics

Education and RINS, Gyeongsang

National University, Jinju,

Gyeongsangnamdo 52828,

Republic of Korea

Full list of author information is

available at the end of the article

\begin{abstract}
In this paper, we consider three types of functions given by products of Bernoulli and Genocchi functions and derive some new identities arising from Fourier series expansions associated with Bernoulli and Genocchi functions. Furthermore, we will express each of them in terms of Bernoulli functions.
\end{abstract}

MSC: $11 \mathrm{~B} 68 ; 11 \mathrm{~B} 83 ; 42 \mathrm{~A} 16$

Keywords: Fourier series; finite product; Bernoulli function; Genocchi function

\section{Introduction}

Utilizing the generating function, the Bernoulli polynomials $B_{m}(x)$ can be written as

$$
\frac{t}{e^{t}-1} e^{x t}=\sum_{m=0}^{\infty} B_{m}(x) \frac{t^{m}}{m !} \quad(\text { see }[1-5])
$$

For $x=0, B_{m}=B_{m}(0)$ are called Bernoulli numbers.

As a second definition, we have the Genocchi polynomials $G_{m}(x)$ by the generating function as follows:

$$
\frac{2 t}{e^{t}+1} e^{x t}=\sum_{m=0}^{\infty} G_{m}(x) \frac{t^{m}}{m !} \quad(\text { see }[6-10]) .
$$

For $x=0, G_{m}=G_{m}(0)$ are called Genocchi numbers.

As to the Bernoulli and Genocchi polynomials and numbers, we will need only the following:

$$
\begin{aligned}
& \frac{d}{d x} B_{m}(x)=m B_{m-1}(x), \quad \frac{d}{d x} G_{m}(x)=m G_{m-1}(x) \quad(m \geq 1), \\
& B_{m}(1)=B_{m}+\delta_{1, m}, \quad G_{m}(1)=-G_{m}+2 \delta_{1, m} \quad(m \geq 0), \\
& G_{0}(x)=0, \quad \operatorname{deg} G_{m}(x)=m-1 \quad(m \geq 1) .
\end{aligned}
$$

For any real number $x$, we let

$$
\langle x\rangle=x-[x] \in[0,1)
$$

denote the fractional part of $x$.

(c) The Author(s) 2017. This article is distributed under the terms of the Creative Commons Attribution 4.0 International License (http://creativecommons.org/licenses/by/4.0/), which permits unrestricted use, distribution, and reproduction in any medium, provided you give appropriate credit to the original author(s) and the source, provide a link to the Creative Commons license, and indicate if changes were made. 
We recall the following facts about the Fourier series expansion of Bernoulli functions $B_{m}(\langle x\rangle)$ :

(a) for $m \geq 2$,

$$
B_{m}(\langle x\rangle)=-m ! \sum_{n=-\infty, n \neq 0}^{\infty} \frac{e^{2 \pi i n x}}{(2 \pi i n)^{m}},
$$

(b) for $m=1$,

$$
-\sum_{n=-\infty, n \neq 0}^{\infty} \frac{e^{2 \pi i n x}}{2 \pi i n}= \begin{cases}B_{1}(\langle x\rangle), & \text { for } x \notin \mathbb{Z}, \\ 0, & \text { for } x \in \mathbb{Z} .\end{cases}
$$

Throughout this paper, we will assume that $r$ and $s$ are nonnegative integers with $r+s \geq 1$. Here we will consider three types of sums of finite products of Bernoulli and Genocchi functions $\alpha_{m}(\langle x\rangle), \beta_{m}(\langle x\rangle)$, and $\gamma_{m}(\langle x\rangle)$ and derive the Fourier series expansions of them. In addition, we will express each of them in terms of Bernoulli functions. We have

(1) $\quad \alpha_{m}(\langle x\rangle)=\sum_{c_{1}+\cdots+c_{r}+j_{1}+\cdots+j_{s}=m} B_{c_{1}}(\langle x\rangle) \cdots B_{c_{r}}(\langle x\rangle) \times G_{j_{1}}(\langle x\rangle) \cdots G_{j_{s}}(\langle x\rangle) \quad(m>s) ;$

(2) $\beta_{m}(\langle x\rangle)=\sum_{c_{1}+\cdots+c_{r}+j_{1}+\cdots+j_{s}=m} \frac{1}{c_{1} ! \cdots c_{r} ! j_{1} ! \cdots j_{s} !}$

$$
\times B_{c_{1}}(\langle x\rangle) \cdots B_{c_{r}}(\langle x\rangle) G_{j_{1}}(\langle x\rangle) \cdots G_{j_{s}}(\langle x\rangle) \quad(m>s)
$$

(3) $\gamma_{m}(\langle x\rangle)=\sum_{c_{1}+\cdots+c_{r}+j_{1}+\cdots+j_{s}=m} \frac{1}{c_{1} \cdots c_{r} j_{1} \cdots j_{s}} B_{c_{1}}(\langle x\rangle) \cdots B_{c_{r}}(\langle x\rangle)$

$$
\times G_{j_{1}}(\langle x\rangle) \cdots G_{j_{s}}(\langle x\rangle) \quad(m \geq r+s, \text { if } r>0 \text { or } m>s, \text { if } r=0) .
$$

Here the sums for (1) and (2) run over all nonnegative integers $c_{1}, \ldots, c_{r}$ and positive integers $j_{1}, \ldots, j_{s}$ with $c_{1}+\cdots+c_{r}+j_{1}+\cdots+j_{s}=m$, and the sum for (3) runs over all positive integers $c_{1}, \ldots, c_{r}, j_{1}, \ldots, j_{s}$ with $c_{1}+\cdots+c_{r}+j_{1}+\cdots+j_{s}=m$.

For elementary facts about Fourier analysis, the reader may refer to any textbook (for example, see [11-13]).

As to $\alpha_{m}(\langle x\rangle)$, we note that the polynomial identity (1.7) follows immediately from Theorems 2.1 and 2.2, which is in turn derived from the Fourier series expansion of $\alpha_{m}(\langle x\rangle)$. We have

$$
\begin{gathered}
\sum_{c_{1}+\cdots+c_{r}+j_{1}+\cdots+j_{s}=m} B_{c_{1}}(x) \cdots B_{c_{r}}(x) G_{j_{1}}(x) \cdots G_{j_{s}}(x) \\
=\frac{1}{m+r+s} \sum_{j=0}^{m}\left(\begin{array}{c}
m+r+s \\
j
\end{array}\right) \Delta_{m-j+1} B_{j}(x),
\end{gathered}
$$


where, for $l>s$,

$$
\begin{aligned}
\Delta_{l}= & \sum_{0 \leq a \leq r, 0 \leq c \leq s, r+s-l \leq a \leq r}\left(\begin{array}{l}
r \\
a
\end{array}\right)\left(\begin{array}{l}
s \\
c
\end{array}\right)(-1)^{c} 2^{s-c} \\
& \times \sum_{c_{1}+\cdots+c_{a}+j_{1}+\cdots+j_{c}=l+a+c-r-s} B_{c_{1}} B_{c_{2}} \cdots B_{c_{a}} G_{j_{1}} \cdots G_{j_{c}} \\
& -\sum_{c_{1}+\cdots+c_{r}+j_{1}+\cdots+j_{s}=l} B_{c_{1}} \cdots B_{c_{r}} G_{j_{1}} \cdots G_{j_{s}} .
\end{aligned}
$$

The obvious polynomial identities can be derived also for $\beta_{m}(\langle x\rangle)$ from Theorems 3.1 and 3.2. It is noteworthy that from the Fourier series expansion of the function $\sum_{k=1}^{m-1} \frac{1}{k(m-k)} B_{k}(\langle x\rangle) B_{m-k}(\langle x\rangle)$ we can derive the Faber-Pandharipande-Zagier identity (see [14-18]) and the Miki identity (see [16-19]). The reader may refer to the recent papers [20-24] for related results.

\section{The function $\alpha_{m}(\langle x\rangle)$}

Let $\alpha_{m}(x)=\sum_{c_{1}+\cdots+c_{r}+j_{1}+\cdots+j_{s}=m} B_{c_{1}}(x) \cdots B_{c_{r}}(x) G_{j_{1}}(x) \cdots G_{j_{s}}(x)(m>s)$, where the sum is over all nonnegative integers $c_{1}, \ldots, c_{r}$ and positive integers $j_{1}, \ldots, j_{s}$ satisfying $c_{1}+\cdots+$ $c_{r}+j_{1}+\cdots+j_{s}=m$. Then we will consider the function

$$
\begin{aligned}
& \alpha_{m}(\langle x\rangle) \\
& \quad=\sum_{c_{1}+\cdots+c_{r}+j_{1}+\cdots+j_{s}=m} B_{c_{1}}(\langle x\rangle) \cdots B_{c_{r}}(\langle x\rangle) G_{j_{1}}(\langle x\rangle) \cdots G_{j_{s}}(\langle x\rangle),
\end{aligned}
$$

defined on $\mathbb{R}$, which is periodic with period 1 .

The Fourier series of $\alpha_{m}(\langle x\rangle)$ is

$$
\sum_{n=-\infty}^{\infty} A_{n}^{(m)} e^{2 \pi i n x}
$$

where

$$
A_{n}^{(m)}=\int_{0}^{1} \alpha_{m}(\langle x\rangle) e^{-2 \pi i n x} d x=\int_{0}^{1} \alpha_{m}(x) e^{-2 \pi i n x} d x .
$$

To proceed, we need to observe the following. We have

$$
\begin{aligned}
\alpha_{m}^{\prime}(x)= & \sum_{c_{1}+\cdots+c_{r}+j_{1}+\cdots+j_{s}=m, c_{1} \geq 1} c_{1} B_{c_{1}-1}(x) B_{c_{2}}(x) \cdots B_{c_{r}}(x) G_{j_{1}}(x) \cdots G_{j_{s}}(x) \\
& +\cdots+\sum_{c_{1}+\cdots+c_{r}+j_{1}+\cdots+j_{s}=m, c_{r} \geq 1} B_{c_{1}}(x) \cdots B_{c_{r-1}} c_{r} B_{c_{r}-1}(x) G_{j_{1}}(x) \cdots G_{j_{s}}(x) \\
& +\sum_{c_{1}+\cdots+c_{r}+j_{1}+\cdots+j_{s}=m, j_{1} \geq 2} B_{c_{1}}(x) \cdots B_{c_{r}}(x) j_{1} G_{j_{1}-1}(x) G_{j_{2}}(x) \cdots G_{j_{s}}(x) \\
& +\cdots+\sum_{c_{1}+\cdots+c_{r}+j_{1}+\cdots+j_{s}=m, j_{s} \geq 2} B_{c_{1}}(x) \cdots B_{c_{r}}(x) G_{j_{1}}(x) \cdots G_{j_{s-1}} j_{s} G_{j_{s}-1}(x) \\
= & \sum_{c_{1}+\cdots+c_{r}+j_{1}+\cdots+j_{s}=m-1}\left(c_{1}+1\right) B_{c_{1}}(x) \cdots B_{c_{r}}(x) G_{j_{1}}(x) \cdots G_{j_{s}}(x)
\end{aligned}
$$




$$
\begin{aligned}
& +\cdots+\sum_{c_{1}+\cdots+c_{r}+j_{1}+\cdots+j_{s}=m-1}\left(c_{r}+1\right) B_{c_{1}}(x) \cdots B_{c_{r}}(x) G_{j_{1}}(x) \cdots G_{j_{s}}(x) \\
& +\sum_{c_{1}+\cdots+c_{r}+j_{1}+\cdots+j_{s}=m-1}\left(j_{1}+1\right) B_{c_{1}}(x) \cdots B_{c_{r}}(x) G_{j_{1}}(x) \cdots G_{j_{s}}(x) \\
& +\cdots+\sum_{c_{1}+\cdots+c_{r}+j_{1}+\cdots+j_{s}=m-1}\left(j_{s}+1\right) B_{c_{1}}(x) \cdots B_{c_{r}}(x) G_{j_{1}}(x) \cdots G_{j_{s}}(x) \\
& =(m+r+s-1) \alpha_{m-1}(x) .
\end{aligned}
$$

From this, we obtain

$$
\left(\frac{\alpha_{m+1}(x)}{m+r+s}\right)^{\prime}=\alpha_{m}(x)
$$

and

$$
\int_{0}^{1} \alpha_{m}(x) d x=\frac{1}{m+r+s}\left(\alpha_{m+1}(1)-\alpha_{m+1}(0)\right)
$$

For $m>s$, we set

$$
\begin{aligned}
\Delta_{m}= & \alpha_{m}(1)-\alpha_{m}(0) \\
= & \sum_{c_{1}+\cdots+c_{r}+j_{1}+\cdots+j_{s}=m}\left(B_{c_{1}}(1) \cdots B_{c_{r}}(1) G_{j_{1}}(1) \cdots G_{j_{s}}(1)-B_{c_{1}} \cdots B_{c_{r}} G_{j_{1}} \cdots G_{j_{s}}\right) \\
= & \sum_{c_{1}+\cdots+c_{r}+j_{1}+\cdots+j_{s}=m}\left(B_{c_{1}}+\delta_{1, c_{1}}\right) \cdots\left(B_{c_{r}}+\delta_{1, c_{r}}\right)\left(-G_{j_{1}}+2 \delta_{1, j_{1}}\right) \cdots\left(-G_{j_{s}}+2 \delta_{1, j_{s}}\right) \\
& -\sum_{c_{1}+\cdots+c_{r}+j_{1}+\cdots+j_{s}=m} B_{c_{1}} \cdots B_{c_{r}} G_{j_{1}} \cdots G_{j_{s}} \\
= & \sum_{0 \leq a \leq r, 0 \leq c \leq s, r+s-m \leq a \leq r}\left(\begin{array}{l}
r \\
a
\end{array}\right)\left(\begin{array}{l}
s \\
c
\end{array}\right)(-1)^{c} 2^{s-c} \\
& \quad \times \sum_{c_{1}+\cdots+c_{a}+j_{1}+\cdots+j_{c}=m+a+c-r-s} B_{c_{1}} \cdots B_{c_{a}} G_{j_{1}} \cdots G_{j_{c}} \\
& \quad \sum_{c_{1}+\cdots+c_{r}+j_{1}+\cdots+j_{s}=m} B_{c_{1}} \cdots B_{c_{r}} G_{j_{1}} \cdots G_{j_{s}} .
\end{aligned}
$$

Note here that the sum over all $c_{1}+\cdots+c_{r}+j_{1}+\cdots+j_{s}=m$ of any term with $a$ of $B_{c_{e}}$, $r-a$ of $\delta_{1, c_{f}}(1 \leq e, f \leq r), c$ of $-G_{j_{u}}$, and $s-c$ of $2 \delta_{1, j_{v}}(1 \leq u, v \leq s)$ all give the same sum

$$
\begin{aligned}
& \sum_{c_{1}+\cdots+c_{r}+j_{1}+\cdots+j_{s}=m} B_{c_{1}} \cdots B_{c_{a}} \delta_{1, c_{a+1}} \cdots \delta_{1, c_{r}}\left(-G_{j_{1}}\right) \cdots\left(-G_{j_{c}}\right) \\
& \quad \times\left(2 \delta_{1, j_{c+1}}\right) \cdots\left(2 \delta_{1, j_{s}}\right) \\
& =\sum_{c_{1}+\cdots+c_{a}+j_{1}+\cdots+j_{c}=m+a+c-r-s}(-1)^{c} 2^{s-c} B_{c_{1}} \cdots B_{c_{a}} G_{j_{1}} \cdots G_{j_{c}},
\end{aligned}
$$

which is not an empty sum as long as $m+a+c-r-s \geq c$. 
We now see that

$$
\alpha_{m}(0)=\alpha_{m}(1) \Longleftrightarrow \Delta_{m}=0
$$

and

$$
\int_{0}^{1} \alpha_{m}(x) d x=\frac{1}{m+r+s} \Delta_{m+1} .
$$

We are now going to determine the Fourier coefficients $A_{n}^{(m)}$.

Case 1: $n \neq 0$. We have

$$
\begin{aligned}
A_{n}^{(m)} & =\int_{0}^{1} \alpha_{m}(x) e^{-2 \pi i n x} d x \\
& =-\frac{1}{2 \pi i n}\left[\alpha_{m}(x) e^{-2 \pi i n x}\right]_{0}^{1}+\frac{1}{2 \pi i n} \int_{0}^{1} \alpha_{m}^{\prime}(x) e^{-2 \pi i n x} d x \\
& =-\frac{1}{2 \pi i n}\left(\alpha_{m}(1)-\alpha_{m}(0)\right)+\frac{m+r+s-1}{2 \pi i n} \int_{0}^{1} \alpha_{m-1}(x) e^{-2 \pi i n x} d x \\
& =\frac{m+r+s-1}{2 \pi i n} A_{n}^{(m-1)}-\frac{1}{2 \pi i n} \Delta_{m} \\
& =\frac{m+r+s-1}{2 \pi i n}\left(\frac{m+r+s-2}{2 \pi i n} A_{n}^{(m-2)}-\frac{1}{2 \pi i n} \Delta_{m-1}\right)-\frac{1}{2 \pi i n} \Delta_{m} \\
& =\frac{(m+r+s-1)_{2}}{(2 \pi i n)^{2}} A_{n}^{(m-2)}-\sum_{j=1}^{2} \frac{(m+r+s-1)_{j-1}}{(2 \pi i n)^{j}} \Delta_{m-j+1} \\
& =\cdots \\
& =\frac{(m+r+s-1)_{m-s}}{(2 \pi i n)^{m-s}} A_{n}^{(s)}-\sum_{j=1}^{m-s} \frac{(m+r+s-1)_{j-1}}{(2 \pi i n)^{j}} \Delta_{m-j+1} \\
= & -\sum_{j=1}^{m-s} \frac{(m+r+s-1)_{j-1}}{(2 \pi i n)^{j}} \Delta_{m-j+1} \cdot
\end{aligned}
$$

Thus we have shown that

$$
A_{n}^{(m)}=-\frac{1}{m+r+s} \sum_{j=1}^{m-s} \frac{(m+r+s)_{j}}{(2 \pi i n)^{j}} \Delta_{m-j+1} .
$$

Case 2: $n=0$. We have

$$
A_{0}^{(m)}=\int_{0}^{1} \alpha_{m}(x) d x=\frac{1}{m+r+s} \Delta_{m+1} .
$$

$\alpha_{m}(\langle x\rangle)(m>s)$ is piecewise $C^{\infty}$. Moreover, $\alpha_{m}(\langle x\rangle)$ is continuous for those integers $m>s$ with $\Delta_{m}=0$, and discontinuous with jump discontinuities at integers for those integers $m>s$ with $\Delta_{m} \neq 0$. 
Assume first that $\Delta_{m}=0$, for an integer $m>s$. Then $\alpha_{m}(0)=\alpha_{m}(1)$.

Hence $\alpha_{m}(\langle x\rangle)$ is piecewise $C^{\infty}$, and continuous. Thus the Fourier series of $\alpha_{m}(\langle x\rangle)$ converges uniformly to $\alpha_{m}(\langle x\rangle)$, and

$$
\begin{aligned}
\alpha_{m}(\langle x\rangle)= & \frac{1}{m+r+s} \Delta_{m+1} \\
& +\sum_{n=-\infty, n \neq 0}^{\infty}\left(-\frac{1}{m+r+s} \sum_{j=1}^{m-s} \frac{(m+r+s)_{j}}{(2 \pi i n)^{j}} \Delta_{m-j+1}\right) e^{2 \pi i n x} \\
= & \frac{1}{m+r+s} \Delta_{m+1}+\frac{1}{m+r+s} \sum_{j=1}^{m-s}\left(\begin{array}{c}
m+r+s \\
j
\end{array}\right) \Delta_{m-j+1} \\
& \times\left(-j ! \sum_{n=-\infty, n \neq 0}^{\infty} \frac{e^{2 \pi i n x}}{(2 \pi i n)^{j}}\right) \\
= & \frac{1}{m+r+s} \Delta_{m+1}+\frac{1}{m+r+s} \sum_{j=2}^{m-s}\left(\begin{array}{c}
m+r+s \\
j
\end{array}\right) \Delta_{m-j+1} B_{j}(\langle x\rangle) \\
& +\Delta_{m} \times \begin{cases}B_{1}(\langle x\rangle), & \text { for } x \notin \mathbb{Z}, \\
0, & \text { for } x \in \mathbb{Z} .\end{cases}
\end{aligned}
$$

We are now ready to state our first result.

Theorem 2.1 For each integer $l>s$, we let

$$
\begin{aligned}
\Delta_{l}= & \sum_{0 \leq a \leq r, 0 \leq c \leq s, r+s-l \leq a \leq r}\left(\begin{array}{l}
r \\
a
\end{array}\right)\left(\begin{array}{l}
s \\
c
\end{array}\right)(-1)^{c} 2^{s-c} \\
& \times \sum_{c_{1}+\cdots+c_{a}+j_{1}+\cdots+j_{c}=l+a+c-r-s} B_{c_{1}} \cdots B_{c_{a}} G_{j_{1}} \cdots G_{j_{c}} \\
& -\sum_{c_{1}+\cdots+c_{r}+j_{1}+\cdots+j_{s}=l} B_{c_{1}} \cdots B_{c_{r}} G_{j_{1}} \cdots G_{j_{s}} .
\end{aligned}
$$

Assume that $\Delta_{m}=0$, for an integer $m>s$. Then we have the following:

(a) $\sum_{c_{1}+\cdots+c_{r}+j_{1}+\cdots+j_{s}=m} B_{c_{1}}(\langle x\rangle) \cdots B_{c_{r}}(\langle x\rangle) G_{j_{1}}(\langle x\rangle) \cdots G_{j_{s}}(\langle x\rangle)$ has the Fourier series expansion

$$
\begin{aligned}
& \sum_{c_{1}+\cdots+c_{r}+j_{1}+\cdots+j_{s}=m} B_{c_{1}}(\langle x\rangle) \cdots B_{c_{r}}(\langle x\rangle) G_{j_{1}}(\langle x\rangle) \cdots G_{j_{s}}(\langle x\rangle) \\
= & \frac{1}{m+r+s} \Delta_{m+1}+\sum_{n=-\infty, n \neq 0}^{\infty}\left(-\frac{1}{m+r+s} \sum_{j=1}^{m-s} \frac{(m+r+s)_{j}}{(2 \pi i n)^{j}} \Delta_{m-j+1}\right) e^{2 \pi i n x}
\end{aligned}
$$

for all $x \in \mathbb{R}$, where the convergence is uniform. 
(b)

$$
\begin{gathered}
\sum_{c_{1}+\cdots+c_{r}+j_{1}+\cdots+j_{s}=m} B_{c_{1}}(\langle x\rangle) \cdots B_{c_{r}}(\langle x\rangle) G_{j_{1}}(\langle x\rangle) \cdots G_{j_{s}}(\langle x\rangle) \\
=\frac{1}{m+r+s} \Delta_{m+1}+\frac{1}{m+r+s} \sum_{j=2}^{m-s}\left(\begin{array}{c}
m+r+s \\
j
\end{array}\right) \Delta_{m-j+1} B_{j}(\langle x\rangle),
\end{gathered}
$$

for all $x \in \mathbb{R}$, where $B_{j}(\langle x\rangle)$ is the Bernoulli function.

Assume next that $\Delta_{m} \neq 0$, for an integer $m>s$. Then $\alpha_{m}(0) \neq \alpha_{m}(1)$. Hence $\alpha_{m}(\langle x\rangle)$ is piecewise $C^{\infty}$, and discontinuous with jump discontinuities at integers. The Fourier series of $\alpha_{m}(\langle x\rangle)$ converges pointwise to $\alpha_{m}(\langle x\rangle)$, for $x \notin \mathbb{Z}$, and converges to

$$
\frac{1}{2}\left(\alpha_{m}(0)+\alpha_{m}(1)\right)=\alpha_{m}(0)+\frac{1}{2} \Delta_{m},
$$

for $x \in \mathbb{Z}$.

Now, we are ready to state our second result.

Theorem 2.2 For each integer $l>s$, we let

$$
\begin{aligned}
\Delta_{l}= & \sum_{0 \leq a \leq r, 0 \leq c \leq s, r+s-l \leq a \leq r}\left(\begin{array}{l}
r \\
a
\end{array}\right)\left(\begin{array}{l}
s \\
c
\end{array}\right)(-1)^{c} 2^{s-c} \\
& \times \sum_{c_{1}+\cdots+c_{a}+j_{1}+\cdots+j_{c}=l+a+c-r-s} B_{c_{1}} B_{c_{2}} \cdots B_{c_{a}} G_{j_{1}} \cdots G_{j_{c}} \\
& -\sum_{c_{1}+\cdots+c_{r}+j_{1}+\cdots+j_{s}=l} B_{c_{1}} \cdots B_{c_{r}} G_{j_{1}} \cdots G_{j_{s}} .
\end{aligned}
$$

Assume that $\Delta_{m} \neq 0$, for an integer $m>s$. Then we have the following:

(a)

$$
\begin{aligned}
& \frac{1}{m+r+s} \Delta_{m+1} \\
& \quad+\sum_{n=-\infty, n \neq 0}^{\infty}\left(-\frac{1}{m+r+s} \sum_{j=1}^{m-s} \frac{(m+r+s)_{j}}{(2 \pi i n)^{j}} \Delta_{m-j+1}\right) e^{2 \pi i n x} \\
& =\left\{\begin{array}{cc}
\sum_{c_{1}+\cdots+c_{r}+j_{1}+\cdots+j_{s}=m} B_{c_{1}}(\langle x\rangle) \cdots B_{c_{r}}(\langle x\rangle) & \text { for } x \notin \mathbb{Z}, \\
\times G_{j_{1}}(\langle x\rangle) \cdots G_{j_{s}}(\langle x\rangle), & \text { for } x \in \mathbb{Z} . \\
\sum_{c_{1}+\cdots+c_{r}+j_{1}+\cdots+j_{s}=m} B_{c_{1}} \cdots B_{c_{r}} G_{j_{1}} \cdots G_{j_{s}}+\frac{1}{2} \Delta_{m},
\end{array}\right.
\end{aligned}
$$

(b)

$$
\begin{aligned}
& \frac{1}{m+r+s} \sum_{j=0}^{m}\left(\begin{array}{c}
m+r+s \\
j
\end{array}\right) \Delta_{m-j+1} B_{j}(\langle x\rangle) \\
& =\sum_{c_{1}+\cdots+c_{r}+j_{1}+\cdots+j_{s}=m} B_{c_{1}}(\langle x\rangle) \cdots B_{c_{r}}(\langle x\rangle) G_{j_{1}}(\langle x\rangle) \cdots G_{j_{s}}(\langle x\rangle), \quad \text { for } x \notin \mathbb{Z},
\end{aligned}
$$




$$
\begin{aligned}
& \frac{1}{m+r+s} \sum_{j=0, j \neq 1}^{m}\left(\begin{array}{c}
m+r+s \\
j
\end{array}\right) \Delta_{m-j+1} B_{j}(\langle x\rangle) \\
& =\sum_{c_{1}+\cdots+c_{r}+j_{1}+\cdots+j_{s}=m} B_{c_{1}} \cdots B_{c_{r}} G_{j_{1}} \cdots G_{j_{s}}+\frac{1}{2} \Delta_{m}, \quad \text { for } x \in \mathbb{Z} .
\end{aligned}
$$

\section{The function $\boldsymbol{\beta}_{m}(\langle x\rangle)$}

Let $\beta_{m}(x)=\sum_{c_{1}+\cdots+c_{r}+j_{1}+\cdots+j_{s}=m} \frac{1}{c_{1} ! \cdots c_{r} ! j_{1} ! \cdots j_{s} !} B_{c_{1}}(x) \cdots B_{c_{r}}(x) G_{j_{1}}(x) \cdots G_{j_{s}}(x)(m>s)$, where the sum is over all nonnegative integers $c_{1}, \ldots, c_{r}$ and positive integers $j_{1}, \ldots, j_{s}$ satisfying $c_{1}+$ $\cdots+c_{r}+j_{1}+\cdots+j_{s}=m$. Then we consider the function

$$
\begin{aligned}
\beta_{m}(\langle x\rangle)= & \sum_{c_{1}+\cdots+c_{r}+j_{1}+\cdots+j_{s}=m} \frac{1}{c_{1} ! \cdots c_{r} ! j_{1} ! \cdots j_{s} !} B_{c_{1}}(\langle x\rangle) \cdots B_{c_{r}}(\langle x\rangle) \\
& \times G_{j_{1}}(\langle x\rangle) \cdots G_{j_{s}}(\langle x\rangle),
\end{aligned}
$$

defined on $\mathbb{R}$, which is periodic with period 1 .

The Fourier series of $\beta_{m}(\langle x\rangle)$ is

$$
\sum_{n=-\infty}^{\infty} B_{n}^{(m)} e^{2 \pi i n x}
$$

where

$$
B_{n}^{(m)}=\int_{0}^{1} \beta_{m}(\langle x\rangle) e^{-2 \pi i n x} d x=\int_{0}^{1} \beta_{m}(x) e^{-2 \pi i n x} d x
$$

To continue further, we need to observe the following:

$$
\begin{aligned}
\beta_{m}^{\prime}(x) & \sum_{c_{1}+\cdots+c_{r}+j_{1}+\cdots+j_{s}=m, c_{1} \geq 1} \frac{1}{\left(c_{1}-1\right) ! c_{2} ! \cdots c_{r} ! j_{1} ! \cdots j_{s} !} B_{c_{1}-1}(x) B_{c_{2}}(x) \cdots B_{c_{r}}(x) \\
& \times G_{j_{1}}(x) \cdots G_{j_{s}}(x) \\
& +\cdots \\
& +\sum_{c_{1}+\cdots+c_{r}+j_{1}+\cdots+j_{s}=m, c_{r} \geq 1} \frac{1}{c_{1} ! \cdots c_{r-1} !\left(c_{r}-1\right) ! j_{1} ! \cdots j_{s} !} B_{c_{1}}(x) \cdots B_{c_{r-1}}(x) B_{c_{r}-1}(x) \\
& \times G_{j_{1}}(x) \cdots G_{j_{s}}(x) \\
& +\sum_{c_{1}+\cdots+c_{r}+j_{1}+\cdots+j_{s}=m, j_{1} \geq 2} \frac{1}{c_{1} ! \cdots c_{r} !\left(j_{1}-1\right) ! j_{2} ! \cdots j_{s} !} B_{c_{1}}(x) \cdots B_{c_{r}}(x) \\
& \times G_{j_{1}-1}(x) G_{j_{2}}(x) \cdots G_{j_{s}}(x) \\
& +\cdots \sum_{c_{1}+\cdots+c_{r}+j_{1}+\cdots+j_{s}=m, j_{s} \geq 2} \frac{1}{c_{1} ! \cdots c_{r} ! j_{1} ! \cdots j_{s-1} !\left(j_{s}-1\right) !} B_{c_{1}}(x) \cdots B_{c_{r}}(x) \\
& \times G_{j_{1}}(x) \cdots G_{j_{s-1}}(x) G_{j_{s}-1}(x)
\end{aligned}
$$




$$
\begin{aligned}
= & \sum_{c_{1}+\cdots+c_{r}+j_{1}+\cdots+j_{s}=m-1} \frac{1}{c_{1} ! \cdots c_{r} ! j_{1} ! \cdots j_{s} !} B_{c_{1}}(x) \cdots B_{c_{r}}(x) G_{j_{1}}(x) \cdots G_{j_{s}}(x) \\
& +\cdots+\sum_{c_{1}+\cdots+c_{r}+j_{1}+\cdots+j_{s}=m-1} \frac{1}{c_{1} ! \cdots c_{r} ! j_{1} ! \cdots j_{s} !} B_{c_{1}}(x) \cdots B_{c_{r}}(x) G_{j_{1}}(x) \cdots G_{j_{s}}(x) \\
& +\sum_{c_{1}+\cdots+c_{r}+j_{1}+\cdots+j_{s}=m-1} \frac{1}{c_{1} ! \cdots c_{r} ! j_{1} ! \cdots j_{s} !} B_{c_{1}}(x) \cdots B_{c_{r}}(x) G_{j_{1}}(x) \cdots G_{j_{s}}(x) \\
& +\cdots+\sum_{c_{1}+\cdots+c_{r}+j_{1}+\cdots+j_{s}=m-1} \frac{1}{c_{1} ! \cdots c_{r} ! j_{1} ! \cdots j_{s} !} B_{c_{1}}(x) \cdots B_{c_{r}}(x) G_{j_{1}}(x) \cdots G_{j_{s}}(x) \\
= & (r+s) \beta_{m-1}(x) .
\end{aligned}
$$

From this, we have

$$
\left(\frac{\beta_{m+1}(x)}{r+s}\right)^{\prime}=\beta_{m}(x)
$$

and

$$
\int_{0}^{1} \beta_{m}(x) d x=\frac{1}{r+s}\left(\beta_{m+1}(1)-\beta_{m+1}(0)\right) .
$$

For $m>s$, we put

$$
\begin{aligned}
& \Omega_{m}=\beta_{m}(1)-\beta_{m}(0) \\
& =\sum_{c_{1}+\cdots+c_{r}+j_{1}+\cdots+j_{s}=m} \frac{1}{c_{1} ! \cdots c_{r} ! j_{1} ! \cdots j_{s} !} B_{c_{1}}(1) \cdots B_{c_{r}}(1) G_{j_{1}}(1) \cdots G_{j_{s}}(1) \\
& -\sum_{c_{1}+\cdots+c_{r}+j_{1}+\cdots+j_{s}=m} \frac{1}{c_{1} ! \cdots c_{r} ! j_{1} ! \cdots j_{s} !} B_{c_{1}} \cdots B_{c_{r}} G_{j_{1}} \cdots G_{j_{s}} \\
& =\sum_{c_{1}+\cdots+c_{r}+j_{1}+\cdots+j_{s}=m} \frac{1}{c_{1} ! \cdots c_{r} ! j_{1} ! \cdots j_{s} !}\left(B_{c_{1}}+\delta_{1, c_{1}}\right) \cdots\left(B_{c_{r}}+\delta_{1, c_{r}}\right) \\
& \times\left(-G_{j_{1}}+2 \delta_{1, j_{1}}\right) \cdots\left(-G_{j_{s}}+2 \delta_{1, j_{s}}\right) \\
& -\sum_{c_{1}+\cdots+c_{r}+j_{1}+\cdots+j_{s}=m} \frac{1}{c_{1} ! \cdots c_{r} ! j_{1} ! \cdots j_{s} !} B_{c_{1}} \cdots B_{c_{r}} G_{j_{1}} \cdots G_{j_{s}} \\
& =\sum_{0 \leq a \leq r, 0 \leq c \leq s, r+s-m \leq a \leq r}\left(\begin{array}{l}
r \\
a
\end{array}\right)\left(\begin{array}{l}
s \\
c
\end{array}\right)(-1)^{c} 2^{s-c} \\
& \times \sum_{c_{1}+\cdots+c_{a}+j_{1}+\cdots+j_{c}=m+a+c-r-s} \frac{1}{c_{1} ! \cdots c_{a} ! j_{1} ! \cdots j_{c} !} B_{c_{1}} \cdots B_{c_{a}} G_{j_{1}} \cdots G_{j_{c}} \\
& -\sum_{c_{1}+\cdots+c_{r}+j_{1}+\cdots+j_{s}=m} \frac{1}{c_{1} ! \cdots c_{r} ! j_{1} ! \cdots j_{s} !} B_{c_{1}} \cdots B_{c_{r}} G_{j_{1}} \cdots G_{j_{s}} . \\
& \beta_{m}(0)=\beta_{m}(1) \quad \Leftrightarrow \quad \Omega_{m}=0,
\end{aligned}
$$


and

$$
\int_{0}^{1} \beta_{m}(x) d x=\frac{1}{r+s} \Omega_{m+1} .
$$

We now would like to determine the Fourier coefficients $B_{n}^{(m)}$.

Case 1: $n \neq 0$. We have

$$
\begin{aligned}
B_{n}^{(m)} & =\int_{0}^{1} \beta_{m}(x) e^{-2 \pi i n x} d x \\
& =-\frac{1}{2 \pi i n}\left[\beta_{m}(x) e^{-2 \pi i n x}\right]_{0}^{1}+\frac{1}{2 \pi i n} \int_{0}^{1} \beta_{m}^{\prime}(x) e^{-2 \pi i n x} d x \\
& =-\frac{1}{2 \pi i n}\left(\beta_{m}(1)-\beta_{m}(0)\right)+\frac{r+s}{2 \pi i n} \int_{0}^{1} \beta_{m-1}(x) e^{-2 \pi i n x} d x \\
& =\frac{r+s}{2 \pi i n} B_{n}^{(m-1)}-\frac{1}{2 \pi i n} \Omega_{m},
\end{aligned}
$$

from which we can deduce that

$$
B_{n}^{(m)}=-\sum_{j=1}^{m-s} \frac{(r+s)^{j-1}}{(2 \pi i n)^{j}} \Omega_{m-j+1} .
$$

Case 2: $n=0$. We have

$$
B_{0}^{(m)}=\int_{0}^{1} \beta_{m}(x) d x=\frac{1}{r+s} \Omega_{m+1} .
$$

$\beta_{m}(\langle x\rangle)(m \geq s)$ is piecewise $C^{\infty}$. Moreover, $\beta_{m}(\langle x\rangle)$ is continuous for those integers $m>s$ with $\Delta_{m}=0$, and discontinuous with jump discontinuities at integers for those integers $m>s$ with $\Delta_{m} \neq 0$.

Assume first that $\Delta_{m}=0$, for an integer $m>s$. Then $\beta_{m}(0)=\beta_{m}(1)$. Hence $\beta_{m}(\langle x\rangle)$ is piecewise $C^{\infty}$, and continuous. Thus the Fourier series of $\beta_{m}(\langle x\rangle)$ converges uniformly to $\beta_{m}(\langle x\rangle)$, and

$$
\begin{aligned}
B_{n}^{(m)}= & \frac{1}{r+s} \Omega_{m+1}+\sum_{n=-\infty, n \neq 0}^{\infty}\left(-\sum_{j=1}^{m-s} \frac{(r+s)^{j-1}}{(2 \pi i n)^{j}} \Omega_{m-j+1}\right) e^{2 \pi i n x} \\
= & \frac{1}{r+s} \Omega_{m+1}+\sum_{j=1}^{m-s} \frac{(r+s)^{j-1}}{j !} \Omega_{m-j+1} \times\left(-j ! \sum_{n=-\infty, n \neq 0}^{\infty} \frac{e^{2 \pi i n x}}{(2 \pi i n)^{j}}\right) \\
= & \frac{1}{r+s} \Omega_{m+1}+\sum_{j=2}^{m-s} \frac{(r+s)^{j-1}}{j !} \Omega_{m-j+1} B_{j}(\langle x\rangle) \\
& +\Omega_{m} \times \begin{cases}B_{1}(\langle x\rangle), & \text { for } x \notin \mathbb{Z}, \\
0, & \text { for } x \in \mathbb{Z} .\end{cases}
\end{aligned}
$$

Now, we are ready to state our first result. 
Theorem 3.1 For each integer $l>s$, we let

$$
\begin{aligned}
\Omega_{l}= & \sum_{0 \leq a \leq r, 0 \leq c \leq s, r+s-l \leq a \leq r}\left(\begin{array}{l}
r \\
a
\end{array}\right)\left(\begin{array}{l}
s \\
c
\end{array}\right)(-1)^{c} 2^{s-c} \\
& \times \sum_{c_{1}+\cdots+c_{a}+j_{1}+\cdots+j_{c}=l+a+c-r-s} \frac{1}{c_{1} ! \cdots c_{a} ! j_{1} ! \cdots j_{c} !} B_{c_{1}} \cdots B_{c_{a}} G_{j_{1}} \cdots G_{j_{c}} \\
& -\sum_{c_{1}+\cdots+c_{r}+j_{1}+\cdots+j_{s}=l} \frac{1}{c_{1} ! \cdots c_{r} ! j_{1} ! \cdots j_{s} !} B_{c_{1}} \cdots B_{c_{r}} G_{j_{1}} \cdots G_{j_{s}} .
\end{aligned}
$$

Assume that $\Omega_{m}=0$, for an integer $m>s$. Then we have the following:

(a) $\sum_{c_{1}+\cdots+c_{r}+j_{1}+\cdots+j_{s}=m} \frac{1}{c_{1} ! \cdots c_{r} ! j_{1} ! \cdots j_{s} !} B_{c_{1}}(\langle x\rangle) \cdots B_{c_{r}}(\langle x\rangle) \times G_{j_{1}}(\langle x\rangle) \cdots G_{j_{s}}(\langle x\rangle)$ has the Fourier series expansion

$$
\begin{aligned}
& \sum_{c_{1}+\cdots+c_{r}+j_{1}+\cdots+j_{s}=m} \frac{1}{c_{1} ! \cdots c_{r} ! j_{1} ! \cdots j_{s} !} B_{c_{1}}(\langle x\rangle) \cdots B_{c_{r}}(\langle x\rangle) \\
& \times G_{j_{1}}(\langle x\rangle) \cdots G_{j_{s}}(\langle x\rangle) \\
& =\frac{1}{r+s} \Omega_{m+1}+\sum_{n=-\infty, n \neq 0}^{\infty}\left(-\sum_{j=1}^{m-s} \frac{(r+s)^{j-1}}{(2 \pi i n)^{j}} \Omega_{m-j+1}\right) e^{2 \pi i n x}
\end{aligned}
$$

for all $x \in \mathbb{R}$, where the convergence is uniform.

(b)

$$
\begin{aligned}
& \sum_{c_{1}+\cdots+c_{r}+j_{1}+\cdots+j_{s}=m} \frac{1}{c_{1} ! \cdots c_{r} ! j_{1} ! \cdots j_{s} !} B_{c_{1}}(\langle x\rangle) \cdots B_{c_{r}}(\langle x\rangle) \\
& \times G_{j_{1}}(\langle x\rangle) \cdots G_{j_{s}}(\langle x\rangle) \\
= & \sum_{j=0, j \neq 1}^{m-s} \frac{(r+s)^{j-1}}{j !} \Omega_{m-j+1} B_{j}(\langle x\rangle)
\end{aligned}
$$

for all $x \in \mathbb{R}$, where $B_{j}(\langle x\rangle)$ is the Bernoulli function.

Assume next that $\Omega_{m} \neq 0$, for an integer $m>s$. Then $\beta_{m}(0) \neq \beta_{m}(1)$. Hence $\beta_{m}(\langle x\rangle)$ is piecewise $C^{\infty}$ and discontinuous with jump discontinuities at integers. The Fourier series of $\beta_{m}(\langle x\rangle)$ converges pointwise to $\beta_{m}(\langle x\rangle)$, for $x \notin \mathbb{Z}$, and converges to

$$
\frac{1}{2}\left(\beta_{m}(0)+\beta_{m}(1)\right)=\beta_{m}(0)+\frac{1}{2} \Omega_{m},
$$

for $x \in \mathbb{Z}$.

We are now ready to state our second result. 
Theorem 3.2 For each integer $l>s$, we let

$$
\begin{aligned}
\Omega_{l}= & \sum_{0 \leq a \leq r, 0 \leq c \leq s, r+s-l \leq a \leq r}\left(\begin{array}{l}
r \\
a
\end{array}\right)\left(\begin{array}{l}
s \\
c
\end{array}\right)(-1)^{c} 2^{s-c} \\
& \times \sum_{c_{1}+\cdots+c_{a}+j_{1}+\cdots+j_{c}=l+a+c-r-s} \frac{1}{c_{1} ! \cdots c_{a} ! j_{1} ! \cdots j_{c} !} B_{c_{1}} \cdots B_{c_{a}} G_{j_{1}} \cdots G_{j_{c}} \\
& -\sum_{c_{1}+\cdots+c_{r}+j_{1}+\cdots+j_{s}=l} \frac{1}{c_{1} ! \cdots c_{r} ! j_{1} ! \cdots j_{s} !} B_{c_{1}} \cdots B_{c_{r}} G_{j_{1}} \cdots G_{j_{s}} .
\end{aligned}
$$

Assume that $\Omega_{m} \neq 0$, for an integer $m>s$. Then we have the following:

(a)

$$
\begin{aligned}
& \frac{1}{r+s} \Omega_{m+1}+\sum_{n=-\infty, n \neq 0}^{\infty}\left(-\sum_{j=1}^{m-s} \frac{(r+s)^{j-1}}{(2 \pi i n)^{j}} \Omega_{m-j+1}\right) e^{2 \pi i n x} \\
& \quad= \begin{cases}\sum_{c_{1}+\cdots+c_{r}+j_{1}+\cdots+j_{s}=m} \frac{1}{c_{1} ! \cdots c_{r} ! j_{1} ! \cdots j_{s} !} B_{c_{1}}(\langle x\rangle) \cdots B_{c_{r}}(\langle x\rangle) & \text { for } x \notin \mathbb{Z}, \\
\times G_{j_{1}}(\langle x\rangle) \cdots G_{j_{s}}(\langle x\rangle), & \text { for } x \in \mathbb{Z} . \\
\sum_{c_{1}+\cdots+c_{r}+j_{1}+\cdots+j_{s}=m} \frac{1}{c_{1} ! \cdots c_{r} ! j_{1} ! \cdots j_{s} !} B_{c_{1}} B_{c_{2}} \cdots B_{c_{r}} G_{j_{1}} \cdots G_{j_{s}}+\frac{1}{2} \Omega_{m}, & \end{cases}
\end{aligned}
$$

(b)

$$
\begin{aligned}
& \sum_{j=0}^{m-s} \frac{(r+s)^{j-1}}{j !} \Omega_{m-j+1} B_{j}(\langle x\rangle) \\
& =\sum_{c_{1}+\cdots+c_{r}+j_{1}+\cdots+j_{s}=m} \frac{1}{c_{1} ! \cdots c_{r} ! j_{1} ! \cdots j_{s} !} B_{c_{1}}(\langle x\rangle) \cdots B_{c_{r}}(\langle x\rangle) \\
& \quad \times G_{j_{1}}(\langle x\rangle) \cdots G_{j_{s}}(\langle x\rangle), \quad \text { for } x \notin \mathbb{Z}, \\
& \sum_{j=0, j \neq 1}^{m-s} \frac{(r+s)^{j-1}}{j !} \Omega_{m-j+1} B_{j}(\langle x\rangle) \\
& =\quad \sum_{c_{1}+\cdots+c_{r}+j_{1}+\cdots+j_{s}=m} \frac{1}{c_{1} ! \cdots c_{r} ! j_{1} ! \cdots j_{s} !} B_{c_{1}} B_{c_{2}} \cdots B_{c_{r}} G_{j_{1}} \cdots G_{j_{s}} \\
& \quad+\frac{1}{2} \Omega_{m}, \quad \text { for } x \in \mathbb{Z} .
\end{aligned}
$$

\section{The function $\gamma_{m}(\langle x\rangle)$}

Here we assume that $r, s$ and $m$ satisfy $m \geq r+s$ if $r>0$ or $m>s$ if $r=0$.

Let $\gamma_{r, s, m}(x)=\sum_{c_{1}+\cdots+c_{r}+j_{1}+\cdots+j_{s}=m} \frac{1}{c_{1} \cdots c_{r} j_{1} \cdots j_{s}} B_{c_{1}}(x) \cdots B_{c_{r}}(x) G_{j_{1}}(x) \cdots G_{j_{s}}(x)$, where the sum is over all positive integers $c_{1}, \ldots, c_{r}, j_{1}, \ldots, j_{s}$ satisfying $c_{1}+\cdots+c_{r}+j_{1}+\cdots+j_{s}=m$. Then we will consider the function

$$
\begin{aligned}
\gamma_{r, s, m}(x)= & \sum_{c_{1}+\cdots+c_{r}+j_{1}+\cdots+j_{s}=m} \frac{1}{c_{1} \cdots c_{r} j_{1} \cdots j_{s}} B_{c_{1}}(\langle x\rangle) \cdots B_{c_{r}}(\langle x\rangle) \\
& \times G_{j_{1}}(\langle x\rangle) \cdots G_{j_{s}}(\langle x\rangle)
\end{aligned}
$$

defined on $\mathbb{R}$, which is periodic with period 1 . 
The Fourier series of $\gamma_{r, s, m}(\langle x\rangle)$ is

$$
\sum_{n=-\infty}^{\infty} C_{n}^{(r, s, m)} e^{2 \pi i n x}
$$

where

$$
C_{n}^{(r, s, m)}=\int_{0}^{1} \gamma_{r, s, m}(\langle x\rangle) e^{-2 \pi i n x} d x=\int_{0}^{1} \gamma_{r, s, m}(x) e^{-2 \pi i n x} d x
$$

To proceed, we need to observe the following. We have

$$
\begin{aligned}
& \gamma_{r, s, m}^{\prime}(x) \\
& =\sum_{c_{1}+\cdots+c_{r}+j_{1}+\cdots+j_{s}=m} \frac{1}{c_{2} \cdots c_{r} j_{1} \cdots j_{s}} B_{c_{1}-1}(x) B_{c_{2}}(x) \cdots B_{c_{r}}(x) G_{j_{1}}(x) \cdots G_{j_{s}}(x) \\
& +\cdots \\
& +\sum_{c_{1}+\cdots+c_{r}+j_{1}+\cdots+j_{s}=m} \frac{1}{c_{1} \cdots c_{r-1} j_{1} \cdots j_{s}} B_{c_{1}}(x) \cdots B_{c_{r-1}}(x) B_{c_{r}-1}(x) G_{j_{1}}(x) \cdots G_{j_{s}}(x) \\
& +\sum_{c_{1}+\cdots+c_{r}+j_{1}+\cdots+j_{s}=m} \frac{1}{c_{1} \cdots c_{r} j_{2} \cdots j_{s}} B_{c_{1}}(x) \cdots B_{c_{r}}(x) G_{j_{1}-1}(x) G_{j_{2}}(x) \cdots G_{j_{s}}(x) \\
& +\cdots \\
& +\sum_{c_{1}+\cdots+c_{r}+j_{1}+\cdots+j_{s}=m} \frac{1}{c_{1} \cdots c_{r} j_{1} \cdots j_{s-1}} B_{c_{1}}(x) \cdots B_{c_{r}}(x) G_{j_{1}}(x) \cdots G_{j_{s-1}}(x) G_{j_{s}-1}(x) \\
& =\sum_{c_{2}+\cdots+c_{r}+j_{1}+\cdots+j_{s}=m-1} \frac{1}{c_{2} \cdots c_{r} j_{1} \cdots j_{s}} B_{c_{2}}(x) \cdots B_{c_{r}}(x) G_{j_{1}}(x) \cdots G_{j_{s}}(x) \\
& +\sum_{c_{1}+\cdots+c_{r}+j_{1}+\cdots+j_{s}=m-1} \frac{1}{c_{2} \cdots c_{r} j_{1} \cdots j_{s}} B_{c_{1}}(x) \cdots B_{c_{r}}(x) G_{j_{1}}(x) \cdots G_{j_{s}}(x) \\
& +\cdots \\
& +\sum_{c_{1}+\cdots+c_{r-1}+j_{1}+\cdots+j_{s}=m-1} \frac{1}{c_{1} \cdots c_{r-1} j_{1} \cdots j_{s}} B_{c_{1}}(x) \cdots B_{c_{r-1}}(x) G_{j_{1}}(x) \cdots G_{j_{s}}(x) \\
& +\sum_{c_{1}+\cdots+c_{r}+j_{1}+\cdots+j_{s}=m-1} \frac{1}{c_{1} \cdots c_{r-1} j_{1} \cdots j_{s}} B_{c_{1}}(x) \cdots B_{c_{r}}(x) G_{j_{1}}(x) \cdots G_{j_{s}}(x) \\
& +\sum_{c_{1}+\cdots+c_{r}+j_{2}+\cdots+j_{s}=m-1} \frac{1}{c_{1} \cdots c_{r} j_{2} \cdots j_{s}} B_{c_{1}}(x) \cdots B_{c_{r}}(x) G_{j_{2}}(x) \cdots G_{j_{s}}(x) \\
& +\sum_{c_{1}+\cdots+c_{r}+j_{1}+\cdots+j_{s}=m-1} \frac{1}{c_{1} \cdots c_{r} j_{2} \cdots j_{s}} B_{c_{1}}(x) \cdots B_{c_{r}}(x) G_{j_{1}}(x) \cdots G_{j_{s}}(x) \\
& +\cdots \\
& +\sum_{c_{1}+\cdots+c_{r}+j_{1}+\cdots+j_{s-1}=m-1} \frac{1}{c_{1} \cdots c_{r} j_{1} \cdots j_{s-1}} B_{c_{1}}(x) \cdots B_{c_{r}}(x) G_{j_{1}}(x) \cdots G_{j_{s-1}}(x)
\end{aligned}
$$




$$
\begin{aligned}
& +\sum_{c_{1}+\cdots+c_{r}+j_{1}+\cdots+j_{s}=m-1} \frac{1}{c_{1} \cdots c_{r} j_{1} \cdots j_{s-1}} B_{c_{1}}(x) \cdots B_{c_{r}}(x) G_{j_{1}}(x) \cdots G_{j_{s}}(x) \\
= & r \gamma_{r-1, s, m-1}(x)+s \gamma_{r, s-1, m-1}(x) \\
& +\sum_{c_{1}+\cdots+c_{r}+j_{1}+\cdots+j_{s}=m-1}\left\{\frac{1}{c_{2} c_{3} \cdots c_{r} j_{1} \cdots j_{s}}+\cdots+\frac{1}{c_{1} \cdots c_{r-1} j_{1} \cdots j_{s}}\right. \\
& \left.+\frac{1}{c_{1} \cdots c_{r} j_{2} \cdots j_{s}}+\cdots+\frac{1}{c_{1} \cdots c_{r} j_{1} \cdots j_{s-1}}\right\} B_{c_{1}}(x) \cdots B_{c_{r}}(x) G_{j_{1}}(x) \cdots G_{j_{s}}(x) \\
= & r \gamma_{r-1, s, m-1}(x)+s \gamma_{r, s-1, m-1}(x)+(m-1) \gamma_{r, s, m-1}(x) .
\end{aligned}
$$

Thus, we have shown that

$$
\gamma_{r, s, m}^{\prime}(x)=r \gamma_{r-1, s, m-1}(x)+s \gamma_{r, s-1, m-1}(x)+(m-1) \gamma_{r, s, m-1}(x) .
$$

Let $m \geq r+s$, for $r>0$, and let $m>s$, for $r=0$. Then we put

$$
\begin{aligned}
\Lambda_{r, s, m}= & \gamma_{r, s, m}(1)-\gamma_{r, s, m}(0) \\
= & \sum_{c_{1}+\cdots+c_{r}+j_{1}+\cdots+j_{s}=m} \frac{1}{c_{1} \cdots c_{r} j_{1} \cdots j_{s}}\left(B_{c_{1}}(1) \cdots B_{c_{r}}(1) G_{j_{1}}(1) \cdots G_{j_{s}}(1)\right. \\
& \left.-B_{c_{1}} \cdots B_{c_{r}} G_{j_{1}} \cdots G_{j_{s}}\right) \\
= & \sum_{c_{1}+\cdots+c_{r}+j_{1}+\cdots+j_{s}=m} \frac{1}{c_{1} \cdots c_{r} j_{1} \cdots j_{s}}\left(\left(B_{c_{1}}+\delta_{1, c_{1}}\right) \cdots\left(B_{c_{r}}+\delta_{1, c_{r}}\right)\right. \\
& \left.\times\left(-G_{j_{1}}+2 \delta_{1, j_{1}}\right) \cdots\left(-G_{j_{s}}+2 \delta_{1, j_{s}}\right)-B_{c_{1}} \cdots B_{c_{r}} G_{j_{1}} \cdots G_{j_{s}}\right) \\
= & \sum_{0 \leq a \leq r, 0 \leq c \leq s}\left(\begin{array}{l}
r \\
a
\end{array}\right)\left(\begin{array}{l}
s \\
c
\end{array}\right)(-1)^{c} 2^{s-c} \\
& \times \sum_{c_{1}+\cdots+c_{a}+j_{1}+\cdots+j_{c}=m+a+c-r-s} \frac{1}{c_{1} \cdots c_{a} j_{1} \cdots j_{c}} B_{c_{1}} \cdots B_{c_{a}} G_{j_{1}} \cdots G_{j_{c}} \\
& -\sum_{c_{1}+\cdots+c_{r}+j_{1}+\cdots+j_{s}=m} \frac{1}{c_{1} \cdots c_{r} j_{1} \cdots j_{s}} B_{c_{1}} \cdots B_{c_{r}} G_{j_{1}} \cdots G_{j_{s}} .
\end{aligned}
$$

From (4.5), we obtain

$$
\gamma_{r, s, m}(x)=-\frac{r}{m} \gamma_{r-1, s, m}(x)-\frac{s}{m} \gamma_{r, s-1, m}(x)+\frac{1}{m} \gamma_{r, s, m+1}^{\prime}(x) .
$$

Denoting $\int_{0}^{1} \gamma_{r, s, m}(x) d x$ by $a_{r, s, m}$, from (4.7) we have

$$
\begin{aligned}
& a_{r, s, m}=-\frac{r}{m} a_{r-1, s, m}-\frac{s}{m} a_{r, s-1, m}+\frac{1}{m} \Lambda_{r, s, m+1}, \\
& a_{r, 0, m}=\int_{0}^{1} \gamma_{r, 0, m}(x) d x=\sum_{j=1}^{r} \frac{(-1)^{j-1}(r)_{j-1}}{m^{j}} \Lambda_{r-j+1,0, m+1} \quad(r \geq 1), \\
& a_{0, s, m}=\int_{0}^{1} \gamma_{0, s, m}(x) d x=\frac{1}{m} \Lambda_{0, s, m+1} \quad(s \geq 1) .
\end{aligned}
$$


Clearly, (4.8) together with (4.9) and (4.10) determines $a_{r, s, m}=\int_{0}^{1} \gamma_{r, s, m}(x) d x$ recursively for all $r, s, m$ satisfying $m \geq r+s$ if $r>0$ or $m>s$ if $r=0$.

Also, we note that

$$
\gamma_{r, s, m}(0)=\gamma_{r, s, m}(1) \quad \Leftrightarrow \quad \Lambda_{r, s, m}=0
$$

We now would like to determine the Fourier coefficients $C_{n}^{(r, s, m)}$.

Case $1: n \neq 0$. We have

$$
\begin{aligned}
& C_{n}^{(r, s, m)}=\int_{0}^{1} \gamma_{r, s, m}(x) e^{-2 \pi i n x} d x \\
& =-\frac{1}{2 \pi i n}\left[\gamma_{r, s, m}(x) e^{-2 \pi i n x}\right]_{0}^{1}+\frac{1}{2 \pi i n} \int_{0}^{1} \gamma_{r, s, m}^{\prime}(x) e^{-2 \pi i n x} d x \\
& =-\frac{1}{2 \pi i n}\left(\gamma_{r, s, m}(1)-\gamma_{r, s, m}(0)\right)+\frac{1}{2 \pi i n} \int_{0}^{1}\left\{r \gamma_{r-1, s, m-1}(x)+s \gamma_{r, s-1, m-1}(x)\right. \\
& \left.+(m-1) \gamma_{r, s, m-1}(x)\right\} e^{-2 \pi i n x} d x \\
& =-\frac{1}{2 \pi i n} \Lambda_{r, s, m}+\frac{1}{2 \pi i n}\left(r C_{n}^{(r-1, s, m-1)}+s C_{n}^{(r, s-1, m-1)}+(m-1) C_{n}^{(r, s, m-1)}\right) \\
& =\frac{m-1}{2 \pi i n} C_{n}^{(r, s, m-1)}+\frac{r}{2 \pi i n} C_{n}^{(r-1, s, m-1)}+\frac{s}{2 \pi i n} C_{n}^{(r, s-1, m-1)}-\frac{1}{2 \pi i n} \Lambda_{r, s, m} \\
& =\frac{m-1}{2 \pi i n}\left(\frac{m-2}{2 \pi i n} C_{n}^{(r, s, m-2)}+\frac{r}{2 \pi i n} C_{n}^{(r-1, s, m-2)}\right. \\
& \left.+\frac{s}{2 \pi i n} C_{n}^{(r, s-1, m-2)}-\frac{1}{2 \pi i n} \Lambda_{r, s, m-1}\right) \\
& +\frac{r}{2 \pi i n} C_{n}^{(r-1, s, m-1)}+\frac{s}{2 \pi i n} C_{n}^{(r, s-1, m-1)}-\frac{1}{2 \pi i n} \Lambda_{r, s, m} \\
& =\frac{(m-1)(m-2)}{(2 \pi i n)^{2}} C_{n}^{(r, s, m-2)}+\frac{r(m-1)}{(2 \pi i n)^{2}} C_{n}^{(r-1, s, m-2)} \\
& +\frac{r}{2 \pi i n} C_{n}^{(r-1, s, m-1)}+\frac{s(m-1)}{(2 \pi i n)^{2}} C_{n}^{(r, s-1, m-2)}+\frac{s}{2 \pi i n} C_{n}^{(r, s-1, m-1)} \\
& -\frac{m-1}{(2 \pi i n)^{2}} \Lambda_{r, s, m-1}-\frac{1}{2 \pi i n} \Lambda_{r, s, m} \\
& =\cdots \\
& =\frac{(m-1)_{m-(r+s)}}{(2 \pi i n)^{m-(r+s)}} C_{n}^{(r, s, r+s)}+\sum_{j=1}^{m-(r+s)} \frac{r(m-1)_{j-1}}{(2 \pi i n)^{j}} C_{n}^{(r-1, s, m-j)} \\
& +\sum_{j=1}^{m-(r+s)} \frac{s(m-1)_{j-1}}{(2 \pi i n)^{j}} C_{n}^{(r, s-1, m-j)}-\sum_{j=1}^{m-(r+s)} \frac{(m-1)_{j-1}}{(2 \pi i n)^{j}} \Lambda_{r, s, m-j+1} \\
& =\sum_{j=1}^{m-(r+s)+1} \frac{r(m-1)_{j-1}}{(2 \pi i n)^{j}} C_{n}^{(r-1, s, m-j)}+\sum_{j=1}^{m-(r+s)} \frac{s(m-1)_{j-1}}{(2 \pi i n)^{j}} C_{n}^{(r, s-1, m-j)} \\
& -\sum_{j=1}^{m-(r+s)+1} \frac{(m-1)_{j-1}}{(2 \pi i n)^{j}} \Lambda_{r, s, m-j+1}
\end{aligned}
$$


Note that

$$
\begin{aligned}
& \frac{(m-1)_{m-(r+s)}}{(2 \pi i n)^{m-(r+s)}} C_{n}^{(r, s, r+s)} \\
& =\frac{(m-1)_{m-(r+s)}}{(2 \pi i n)^{m-(r+s)}} \int_{0}^{1}\left(x-\frac{1}{2}\right)^{r} d x \\
& =\frac{(m-1)_{m-(r+s)}}{(2 \pi i n)^{m-(r+s)}}\left\{-\frac{1}{2 \pi i n}\left(\left(\frac{1}{2}\right)^{r}-\left(-\frac{1}{2}\right)^{r}\right)\right. \\
& \left.\quad+\frac{r}{2 \pi i n} \int_{0}^{1}\left(x-\frac{1}{2}\right)^{r-1} e^{-2 \pi i n x} d x\right\}, \\
& \frac{r(m-1)_{m-(r+s)}}{(2 \pi i n)^{m-(r+s)+1}} C_{n}^{(r-1, s, r+s-1)}=\frac{r(m-1)_{m-(r+s)}}{(2 \pi i n)^{m-(r+s)+1}} \int_{0}^{1}\left(x-\frac{1}{2}\right)^{r-1} e^{-2 \pi i n x} d x, \\
& -\frac{(m-1)_{m-(r+s)}}{(2 \pi i n)^{m-(r+s)+1}} \Lambda_{r, s, r+s}=-\frac{(m-1)_{m-(r+s)}}{(2 \pi i n)^{m-(r+s)+1}}\left(\left(\frac{1}{2}\right)^{r}-\left(-\frac{1}{2}\right)^{r}\right) .
\end{aligned}
$$

Thus we have shown that

$$
\begin{aligned}
C_{n}^{(r, s, m)}= & \sum_{j=1}^{m-(r+s)+1} \frac{r(m-1)_{j-1}}{(2 \pi i n)^{j}} C_{n}^{(r-1, s, m-j)}+\sum_{j=1}^{m-(r+s)} \frac{s(m-1)_{j-1}}{(2 \pi i n)^{j}} C_{n}^{(r, s-1, m-j)} \\
& -\sum_{j=1}^{m-(r+s)+1} \frac{(m-1)_{j-1}}{(2 \pi i n)^{j}} \Lambda_{r, s, m-j+1}, \\
C_{n}^{(r, 0, m)}= & \sum_{j=1}^{m-r+1} \frac{r(m-1)_{j-1}}{(2 \pi i n)^{j}} C_{n}^{(r-1,0, m-j)}-\sum_{j=1}^{m-r+1} \frac{(m-1)_{j-1}}{(2 \pi i n)^{j}} \Lambda_{r, 0, m-j+1} \quad(r \geq 2), \\
C_{n}^{(1,0, m)}= & -\frac{(m-1) !}{(2 \pi i n)^{m}}, \\
C_{n}^{(0, s, m)}= & -\frac{1}{m} \sum_{j=1}^{m-s} \frac{(m)_{j}}{(2 \pi i n)^{j}} \Lambda_{0, s, m-j+1} \quad(s \geq 1) .
\end{aligned}
$$

We now see that $C_{n}^{(r, s, m)}(n \neq 0)$ can be completely determined by (4.14)-(4.17), for all $r$, $s, m$ satisfying $m \geq r+s$ if $r>0$ or $m>s$ if $r=0$.

Case 2: $n=0$. We have

$$
C_{0}^{(r, s, m)}=\int_{0}^{1} \gamma_{r, s, m}(x) d x
$$

which can be determined from (4.8)-(4.10), for all $r, s, m$ satisfying $m \geq r+s$ if $r>0$ or $m>s$ if $r=0$.

$\gamma_{r, s, m}(\langle x\rangle)$ is piecewise $C^{\infty}$. Moreover, $\gamma_{r, s, m}(\langle x\rangle)$ is continuous for those integers $r, s, m$ with $\Lambda_{r, s, m}=0$ and discontinuous with jump discontinuities at integers for those integers $r, s, m$ with $\Lambda_{r, s, m} \neq 0$.

Assume first that $\Lambda_{r, s, m}=0$, for some integers $r, s, m$ satisfying $m \geq r+s$ if $r>0$ or $m>s$ if $r=0$. Then $\gamma_{r, s, m}(0)=\gamma_{r, s, m}(1)$. Thus $\gamma_{r, s, m}(\langle x\rangle)$ is piecewise $C^{\infty}$ and continuous. Hence 
the Fourier series of $\gamma_{r, s, m}(\langle x\rangle)$ converges uniformly to $\gamma_{r, s, m}(\langle x\rangle)$, and

$$
\gamma_{r, s, m}(\langle x\rangle)=C_{0}^{(r, s, m)}+\sum_{n=-\infty, n \neq 0}^{\infty} C_{n}^{(r, s, m)} e^{2 \pi i n x},
$$

where $C_{0}^{(r, s, m)}$ are determined by (4.8)-(4.10) and $C_{n}^{(r, s, m)}(n \neq 0)$ by (4.14)-(4.17).

We are now going to state our first result.

Theorem 4.1 For all integers $r, s$, $l$ satisfying $l \geq r+s$, for $r>0$ or $l>s$, for $r=0$, we let

$$
\begin{aligned}
\Lambda_{r, s, l}= & \sum_{0 \leq a \leq r, 0 \leq c \leq s}\left(\begin{array}{l}
r \\
a
\end{array}\right)\left(\begin{array}{l}
s \\
c
\end{array}\right)(-1)^{c} 2^{s-c} \\
& \times \sum_{c_{1}+\cdots+c_{a}+j_{1}+\cdots+j_{c}=l+a+c-r-s} \frac{1}{c_{1} \cdots c_{a} j_{1} \cdots j_{c}} B_{c_{1}} \cdots B_{c_{a}} G_{j_{1}} \cdots G_{j_{c}} \\
& -\sum_{c_{1}+\cdots+c_{r}+j_{1}+\cdots+j_{s}=l} \frac{1}{c_{1} \cdots c_{r} j_{1} \cdots j_{s}} B_{c_{1}} \cdots B_{c_{r}} G_{j_{1}} \cdots G_{j_{s}} .
\end{aligned}
$$

Assume that $\Lambda_{r, s, m}=0$, for some integers $r, s, m$ satisfying $m \geq r+s$ if $r>0$ or $m>s$ if $r=0$. Then we have the following:

$$
\begin{aligned}
& \sum_{c_{1}+\cdots+c_{r}+j_{1}+\cdots+j_{s}=m} \frac{1}{c_{1} \cdots c_{r} j_{1} \cdots j_{s}} B_{c_{1}}(\langle x\rangle) \cdots B_{c_{r}}(\langle x\rangle) \\
& \times G_{j_{1}}(\langle x\rangle) \cdots G_{j_{s}}(\langle x\rangle)
\end{aligned}
$$

has the Fourier series expansion

$$
\begin{aligned}
& \sum_{c_{1}+\cdots+c_{r}+j_{1}+\cdots+j_{s}=m} \frac{1}{c_{1} \cdots c_{r} j_{1} \cdots j_{s}} B_{c_{1}}(\langle x\rangle) \cdots B_{c_{r}}(\langle x\rangle) \\
& \times G_{j_{1}}(\langle x\rangle) \cdots G_{j_{s}}(\langle x\rangle) \\
= & C_{0}^{(r, s, m)}+\sum_{n=-\infty, n \neq 0}^{\infty} C_{n}^{(r, s, m)} e^{2 \pi i n x},
\end{aligned}
$$

where $C_{n}^{(r, s, m)}(n \neq 0)$ are determined by (4.14)-(4.17) and $C_{0}^{(r, s, m)}$ by (4.8)-(4.10). Here the convergence is uniform.

Next, assume that $\Lambda_{r, s, m} \neq 0$, for some integers satisfying $m \geq r+s$ if $r>0$ or $m>s$ if $r=0$. Then $\gamma_{r, s, m}(0) \neq \gamma_{r, s, m}(1)$. Here $\gamma_{r, s, m}(\langle x\rangle)$ is piecewise $C^{\infty}$ and discontinuous with jump discontinuities at integers. Then the Fourier series of $\gamma_{r, s, m}(\langle x\rangle)$ converges pointwise to $\gamma_{r, s, m}(\langle x\rangle)$, for $x \notin \mathbb{Z}$, and it converges to

$$
\frac{1}{2}\left(\gamma_{r, s, m}(0)+\gamma_{r, s, m}(1)\right)=\gamma_{r, s, m}(0)+\frac{1}{2} \Lambda_{r, s, m},
$$

for $x \in \mathbb{Z}$.

Now, we are going to state our second result. 
Theorem 4.2 For all integers $r, s, l$ satisfying $l \geq r+s$, for $r>0$ or $l>s$, for $r=0$, we let

$$
\begin{aligned}
\Lambda_{r, s, l}= & \sum_{0 \leq a \leq r, 0 \leq c \leq s}\left(\begin{array}{l}
r \\
a
\end{array}\right)\left(\begin{array}{l}
s \\
c
\end{array}\right)(-1)^{c} 2^{s-c} \\
& \times \sum_{c_{1}+\cdots+c_{a}+j_{1}+\cdots+j_{c}=l+a+c-r-s} \frac{1}{c_{1} \cdots c_{a} j_{1} \cdots j_{c}} B_{c_{1}} \cdots B_{c_{a}} G_{j_{1}} \cdots G_{j_{c}} \\
& -\sum_{c_{1}+\cdots+c_{r}+j_{1}+\cdots+j_{s}=l} \frac{1}{c_{1} \cdots c_{r} j_{1} \cdots j_{s}} B_{c_{1}} \cdots B_{c_{r}} G_{j_{1}} \cdots G_{j_{s}} .
\end{aligned}
$$

Assume that $\Lambda_{r, s, m} \neq 0$, for some integers $r, s, m$ satisfying $m \geq r+s$ if $r>0$ or $m>s$ if $r=0$. Then we have the following:

$$
\begin{aligned}
& C_{0}^{(r, s, m)}+\sum_{n=-\infty, n \neq 0}^{\infty} C_{n}^{(r, s, m)} e^{2 \pi i n x} \\
& \quad= \begin{cases}\sum_{c_{1}+\cdots+c_{r}+j_{1}+\cdots+j_{s}=m} \frac{1}{c_{1} \cdots c_{r} j_{1} \cdots j_{s}} B_{c_{1}}(\langle x\rangle) \cdots B_{c_{r}}(\langle x\rangle) & \text { for } x \notin \mathbb{Z}, \\
\quad \times G_{j_{1}}(\langle x\rangle) \cdots G_{j_{s}}(\langle x\rangle), & \text { for } x \in \mathbb{Z}, \\
\sum_{c_{1}+\cdots+c_{r}+j_{1}+\cdots+j_{s}=m} \frac{1}{c_{1} \cdots c_{r} j_{1} \cdots j_{s}} B_{c_{1}} \cdots B_{c_{r}} G_{j_{1}} \cdots G_{j_{s}}+\frac{1}{2} \Lambda_{r, s, m},\end{cases}
\end{aligned}
$$

where $C_{n}^{(r, s, m)}(n \neq 0)$ are determined by (4.14)-(4.17) and $C_{0}^{(r, s, m)}$ by (4.8)-(4.10).

\section{Results and discussion}

In this paper, we study three types of functions which are given by products of Bernoulli and Genocchi functions and we give some new identities arising from Fourier series expansions associated with Bernoulli and Genocchi functions. In addition, we will express each of them in terms of Bernoulli functions. The Fourier series expansion of the Bernoulli and Genocchi functions are useful in computing the special values of the zeta and multiple zeta function. It is expected that the Fourier series of the Bernoulli and Genocchi functions will find some applications in connection with a certain zeta function and the higher-order Bernoulli numbers.

\section{Conclusion}

In this paper, we considered the Fourier series expansion of the Bernoulli and Genocchi functions which are obtained by extending by periodicity of period the Bernoulli and Genocchi polynomials on $[0,1)$. The Fourier series are explicitly determined.

\section{Acknowledgements}

The first author has been appointed a chair as professor at Tianjin Polytechnic University by Tianjin City in China from August 2015 to August 2019.

\section{Competing interests}

The authors declare that they have no competing interests.

\section{Authors' contributions}

All authors contributed equally to the manuscript and typed, read, and approved the final manuscript.

\section{Author details}

'Department of Mathematics, Kwangwoon University, Seoul, 139-701, Republic of Korea. ${ }^{2}$ Department of Mathematics, College of Science, Tianjin Polytechnic University, Tianjin, 300160, China. ${ }^{3}$ Department of Mathematics, Sogang University, Seoul, 121-742, Republic of Korea. ${ }^{4}$ Department of Mathematics Education and RINS, Gyeongsang National University, Jinju, Gyeongsangnamdo 52828, Republic of Korea. 


\section{Publisher's Note}

Springer Nature remains neutral with regard to jurisdictional claims in published maps and institutional affiliations.

Received: 10 May 2017 Accepted: 5 June 2017 Published online: 30 June 2017

\section{References}

1. Faber, C, Pandharipande, R: Hodge integrals and Gromov-Witten theory. Invent. Math. 139(1), 173-199 (2000)

2. Kim, DS, Kim, T: A note on higher-order Bernoulli polynomials. J. Inequal. Appl. 2013, 111 (2013)

3. Kim, DS, Kim, T: Bernoulli basis and the product of several Bernoulli polynomials. Int. J. Math. Math. Sci. 2012, 463659 (2012)

4. Kim, T: Some identities for the Bernoulli, the Euler and Genocchi numbers and polynomials. Adv. Stud. Contemp. Math. 20(1), 23-28 (2015)

5. Liu, H, Wang, W: Some identities on the the Bernoulli, Euler and Genocchi poloynomials via power sums and alternate power sums. Discrete Math. 309, 3346-3363 (2009)

6. Cangul, IN, Kurt, V, Ozden, H, Simsek, Y: On the higher-order w-q-Genocchi numbers. Adv. Stud. Contemp. Math. (Kyungshang) 19(1), 39-57 (2009)

7. Ding, D, Yang, J: Some identities related to the Apostol-Euler and Apostol-Bernoulli polynomials. Adv. Stud. Contemp. Math. (Kyungshang) 20(1), 7-21 (2010)

8. Dunne, GV, Schubert, C: Bernoulli number identities from quantum field theory and topological string theory. Commun. Number Theory Phys. 7(2), 225-249 (2013)

9. Luo, QM: Fouier expansions and integral representations for Genocchi poloynomials. J. Integer Seq. 12, 09.1.4 (2009)

10. Srivastava, HM: Some generalizations and basic extensions of the Bernoulli, Euler and Genocchi polynomials. Appl. Math. Inf. Sci. 5(3), 390-414 (2011)

11. Abramowitz, M, Stegun, IA: Handbook of Mathematical Functions. Dover, New York (1970)

12. Marsden, JE: Elementary Classical Analysis. Freeman, New York (1974)

13. Zill, DG, Cullen, MR: Advanced Engineering Mathematics. Jones \& Bartlett, Boston (2006)

14. Gaboury, S, Tremblay, R, Fugere, B-J: Some explicit formulas for certain new classes of Bernoulli, Euler and Genocchi polynomials. Proc. Jangjeon Math. Soc. 17(1), 115-123 (2014)

15. Jang, G-W, Kim, T, Kim, DS, Mansour, T: Fourier series of functions related to Bernoulli polynomials. Adv. Stud. Contemp. Math. 27, 49-62 (2017)

16. Kim, DS, Kim, T: Some identities of higher order Euler polynomials arising from Euler basis. Integral Transforms Spec. Funct. 24(9), 734-738 (2013)

17. Kim, T: On the multiple $q-G e n o c c h i$ and Euler numbers. Russ. J. Math. Phys. 15(4), 481-486 (2008)

18. Kim, T: Euler numbers and polynomials associated with zeta functions. Abstr. Appl. Anal. 2008, Article ID 581582 (2008)

19. Miki, H: A relation between Bernoulli numbers. J. Number Theory 10(3), 297-302 (1978)

20. Kim, DS, Kim, T: Identities arising from higher-order Daehee polynomial bases. Open Math. 13, 196-208 (2015)

21. Kim, T, Kim, DS, Dolgy, D, Park, J-W: Fourier series of sums of products of poly-Bernoulli functions and their applications. J. Nonlinear Sci. Appl. 10(4), 2384-2401 (2017)

22. Kim, T, Kim, DS, Dolgy, D, Park, J-W: Fourier series of sums of products of ordered Bell and poly-Bernoulli functions. J. Inequal. Appl. 2017, 13660 (2017)

23. Kim, T, Kim, DS, Jang, G-W, Kwon, J: Fourier series of sums of products of Genocchi functions and their applications. J. Nonlinear Sci. Appl. 10(4), 1683-1694 (2017)

24. Kim, T, Kim, DS, Rim, S-H, Dolgy, D: Fourier series of higher-order Bernoulli functions and their applications. J. Inequal. Appl. 2017, $71452(2017)$

\section{Submit your manuscript to a SpringerOpen ${ }^{\circ}$ journal and benefit from:}

- Convenient online submission

- Rigorous peer review

Open access: articles freely available online

- High visibility within the field

- Retaining the copyright to your article

Submit your next manuscript at $\gg$ springeropen.com 